\title{
Velocidad de procesamiento en la esclerosis múltiple remitente recurrente: el papel de los síntomas depresivos
}

\author{
Genny Lubrini, José Antonio Periáñez, Marcos Ríos-Lago, Ana Frank
}

Introducción. La disminución de la velocidad de procesamiento parece ser una característica neuropsicológica prototípica en la esclerosis múltiple (EM). Sin embargo, el impacto de los frecuentes síntomas depresivos de estos pacientes sobre la velocidad de procesamiento aún no se ha definido con precisión, debido a algunas limitaciones metodológicas presentes en la mayoría de los estudios previos.

Sujetos y métodos. Cuarenta y dos pacientes con EM remitente recurrente, 20 con síntomas depresivos (inventario de depresión de Beck > 13) y 22 sin ellos, fueron comparados con 24 controles sanos en tests neuropsicológicos de velocidad de procesamiento -Symbol Digit Modality Test (SDMT), test de Stroop, Trail Making Test (TMT) y Paced Auditory Serial Addition Test-batería neuropsicológica breve (PASAT-BNB)-.

Resultados. Los pacientes con EM sin síntomas depresivos realizaron significativamente peor que los controles los tests de Stroop - palabra (P), color (C) y palabra-color (PC) - y PASAT-BNB (tiempo de ejecución), mientras que los pacientes con síntomas depresivos obtuvieron puntuaciones significativamente peores que los controles sanos en Stroop $(P, C$ y $P C)$, SDMT, TMT ( $A$, B y B-A) y PASAT-BNB (tiempo de ejecución y errores). La ejecución de los pacientes con EM y síntomas depresivos fue significativamente peor que la de los pacientes sin síntomas depresivos en el SDMT, TMT (A, B y B-A) y PASAT-BNB (tiempo de ejecución).

Conclusiones. El impacto de la enfermedad no se evidenció de forma generalizada en el rendimiento neuropsicológico de los pacientes con EM remitente recurrente. Los síntomas depresivos parecen desempeñar un papel importante en la determinación de los déficits de velocidad. Mientras que la EM redujo la velocidad de procesamiento, los síntomas depresivos en la EM se asociaron a un déficit específico en ciertos procesos cognitivos diferentes de los de velocidad.

Palabras clave. Atención. Depresión. Esclerosis múltiple. Evaluación neuropsicológica. Funciones ejecutivas. Velocidad de procesamiento.

\section{Introducción}

El estudio de las alteraciones cognitivas asociadas a la esclerosis múltiple (EM) ha mostrado la presencia de déficits en distintos dominios cognitivos, compatibles con la naturaleza multifocal de la enfermedad [1-3]. Entre éstos destaca la disminución de la velocidad de procesamiento de la información (VP) constatada mediante diferentes instrumentos, como el Paced Auditory Serial Addition Test (PASAT) [4,5], el Symbol Digit Modality Test (SDMT) [6,7], el test de Stroop [8,9], la torre de Londres [3,9] y el Trail Making Test (TMT) [10]. Muchas investigaciones en EM han intentado establecer la relación entre dichos déficits de velocidad y otras variables clínicas, como, por ejemplo, la presencia de alteraciones neurológicas, la duración de la enfermedad o la presencia de síntomas neuropsiquiátricos. Entre estos últimos cabe destacar la importancia de la depresión, dado que el 27-54\% de los pacientes con EM presenta síntomas depresivos $[11,12]$, los cua- les podrían modular las alteraciones de velocidad, al igual que en la población general $[13,14]$.

La investigación neuropsicológica sobre VP y depresión en EM hasta la fecha se ha desarrollado en dos sentidos. Por un lado, un importante grupo de trabajos ha tratado de controlar el potencial efecto de la depresión mediante análisis de covarianza o mediante la equiparación entre grupos de los síntomas depresivos. Los resultados han mostrado que la depresión no parece responsable del pobre rendimiento de los pacientes con EM en comparación con los controles en tareas neuropsicológicas [15-18], y que los déficits de velocidad parecen el principal mecanismo responsable de las diferencias [6,16-18]. Denney et al [16], por ejemplo, mostraron que los pacientes con EM realizaron el test de Stroop y la torre de Londres peor que los controles. Además, el estudio de covarianza reveló que las diferencias entre los grupos no desaparecieron al controlar el efecto de la depresión mediante el cuestionario de depresión Center for Epidemiolo-
Servicio de Neurología; Unidad de Trastornos Cognitivos; Hospital Universitario La Paz; Instituto de Investigación Biomédica (IdiPAZ); Universidad Autónoma de Madrid (G. Lubrini, A. Frank). Departamento de Psicología Básica II; Universidad Complutense de Madrid (G. Lubrini, J.A. Periáñez). Unidad de Investigación Proyecto Alzheimer; Fundación CIEN; Fundación Reina Sofía (J.A. Periáñez, M. Ríos-Lago). Red Menni de Daño Cerebral; Hermanas Hospitalarias (M. Ríos-Lago). Departamento de Psicología Básica II; Universidad Nacional de Educación a Distancia (M. Ríos-Lago). Madrid, España.

Correspondencia:

Dra. Genny Lubrini. Servicio de Neurología. Hospital Universitario La Paz. Instituto de Investigación Biomédica (IdiPAZ). Universidad Autónoma de Madrid. Paseo de la Castellana, 261. E-28046 Madrid.

Fax:

+34 917277324.

E-mail:

genny.lubrini@hotmail.it

Financiación:

Parcialmente financiado por el Ministerio de Ciencia e Innovación (PSI2009-14415-C03-03) y por Merck-Serono.

Aceptado tras revisión externa: 13.09.12.

Cómo citar este artículo: Lubrini G, Periáñez JA, Ríos-Lago M, Frank A. Velocidad de procesamiento en la esclerosis múltiple remitente recurrente: el papel de los síntomas depresivos. Rev Neurol 2012; 55 : 585-92.

(c) 2012 Revista de Neurología 
gic Studies-Depression Scale [19]. Estos y otros hallazgos han llevado a la formalización de la hipótesis de la VP en la EM (relative consequence model [20]), según la cual la disminución de la VP es el mecanismo que está en la base del bajo rendimiento de los pacientes con EM en los tests neuropsicológicos [5,21-25]. Sin embargo, el empleo de las puntuaciones de los cuestionarios de depresión como una covariable parece resultar mínimamente eficaz cuando se trata de describir la relación entre deterioro cognitivo y depresión [26-28]. Siendo la relación entre síntomas depresivos y funcionamiento cognitivo no lineal, algunos han señalado que resultaría necesario que los síntomas depresivos alcanzaran un umbral de gravedad suficiente para que el déficit se haga evidente [29]. En consecuencia, la aparente ausencia de relación entre depresión y VP en este tipo de trabajos podría deberse a las limitaciones de las técnicas de control estadístico empleadas, más que a una verdadera independencia entre estas dos variables.

Salvando esta dificultad, un segundo grupo de investigaciones ha considerado la depresión como variable de agrupación. Los resultados de estos estudios han venido demostrando que los pacientes con EM sin depresión no muestran diferencias con los controles en tests como el SDMT [26], el PASAT (dos y tres segundos) [26] o el test de Stroop [16]. Sin embargo, los pacientes con EM deprimidos realizaron significativamente peor que los pacientes con EM no deprimidos tests como la torre de Londres [3,30], el SDMT (versión oral) [26], el PASAT [26] y el Visual Threshold Serial Addition Test [31]. En conjunto, las evidencias de estas investigaciones sugieren que el impacto de la EM por sí sola no se evidencia de forma generalizada en todos los tests neuropsicológicos, y que la depresión podría desempeñar un papel importante en determinar los problemas de velocidad de los pacientes con EM. Sin embargo, la mayoría de estos estudios no ha profundizado en analizar la influencia de la VP en la ejecución de los tests neuropsicológicos de los pacientes.

Cabe señalar que, a día de hoy, resultan escasas las investigaciones que hayan tratado de analizar el rendimiento neuropsicológico de pacientes con diferentes subtipos de EM, pese a la constatación de diferencias en la gravedad de los déficits de velocidad dependiendo del curso clínico de la enfermedad [32]. Más concretamente, los pacientes con EM remitente recurrente (EMRR) parecen ser más lentos que los controles sanos, pero más rápidos que los pacientes con EM primaria progresiva y secundaria progresiva $[4,16,30]$. Así, la heterogeneidad de las muestras empleadas en estudios previos podría di- ficultar la interpretación de los resultados neuropsicológicos, aumentando el riesgo de subestimar o magnificar los déficits de velocidad característicos de un subtipo específico de EM.

\section{Objetivos}

El objetivo del presente trabajo de investigación se centró en determinar el impacto de la EM en la realización de tests neuropsicológicos sensibles a los déficits de velocidad en pacientes con y sin síntomas depresivos. Con este fin, se comparó el rendimiento neuropsicológico de un grupo homogéneo de pacientes con EMRR sin síntomas depresivos con el de un grupo de participantes sanos. La influencia de los síntomas depresivos en el rendimiento de los pacientes con EM se evaluó mediante la comparación del rendimiento entre pacientes con EM con y sin síntomas depresivos. Por último, y para clarificar si la disminución de la VP constituye la alteración subyacente a las alteraciones cognitivas de los pacientes con EM, tal cual predice la hipótesis de la VP (relative consequence model [20]), se incluyeron en la batería de evaluación empleada índices neuropsicológicos que han demostrado capacidad para aislar las alteraciones de la VP de las de otras variables cognitivas implicadas en la realización de dichos tests.

\section{Sujetos y métodos}

Cuarenta y dos pacientes con EM de la Unidad de Neuroinmunología y Esclerosis Múltiple del Servicio de Neurología del Hospital Universitario La Paz de Madrid fueron seleccionados para este estudio de acuerdo con los siguientes criterios de inclusión: diagnóstico de EMRR según los criterios de McDonald [33]; puntuación < 6 en la Expanded Disability Status Scale (EDSS) [34]; en caso de brote reciente se requería que hubiera transcurrido al menos un mes desde la remisión de los síntomas; y no haber recibido tratamiento con esteroides en el mes previo a la evaluación. Los pacientes con EM fueron asignados a dos grupos a partir de sus puntuaciones en la versión española del inventario de depresión de Beck (BDI) [35,36]. Resultado de ello, 22 pacientes con EM fueron asignados al grupo de pacientes con síntomas depresivos y 20 al grupo de pacientes sin síntomas depresivos, estableciendo el punto de corte para el grupo con síntomas depresivos en la puntuación BDI > 13 [10,37]. Veinticuatro sujetos control equiparados en género, edad y nivel 
educativo con ambos grupos de pacientes reclutados mediante anuncios en el hospital y en la universidad también participaron en el estudio. Los participantes sanos no presentaron síntomas depresivos (puntuación BDI < 13 en todos los casos). Los criterios de exclusión para los 66 participantes fueron: presencia de antecedentes de traumatismo craneoencefálico, accidentes cerebrovasculares u otras enfermedades del sistema nervioso central, presencia de abuso de alcohol u otras drogas (excluida nicotina), y presencia de trastornos visuales no corregidos o motores que pudieran interferir en la adecuada realización de las pruebas.

Todos los participantes eran diestros [38]. Las puntuaciones en el Stroop, TMT y PASAT-BNB no pudieron registrarse en todos los participantes del grupo EM con síntomas depresivos, mientras que las puntuaciones del Stroop no pudieron registrarse en un participante del grupo EM sin síntomas depresivos debido al extravío de los protocolos. Pese a ello, y dado el carácter independiente de las comparaciones estadísticas realizadas entre las puntuaciones de los tests neuropsicológicos seleccionados, ninguno de estos participantes fue eliminado de los análisis. La tabla I especifica las características demográficas y clínicas de las muestras comparadas en cada caso. Los participantes fueron informados de los detalles de la investigación y manifestaron por escrito su consentimiento a participar en el estudio.

La evaluación neuropsicológica de los participantes se llevó a cabo en una única sesión de aproximadamente 45 minutos de duración entre las 9 y las 12 de la mañana, e incluyó los siguientes tests: SDMT [39] para la evaluación de la VP mediante el cómputo del número de dígitos correctamente asociados a los símbolos en un tiempo de 90 segundos; test de colores y palabras (Stroop) [40] para evaluar la capacidad de control de la interferencia y la VP mediante el registro del número de ítems completados en 45 segundos en cada una de las tres condiciones -Stroop palabra (P), Stroop color (C) y Stroop palabra-color (P-C) - y la puntuación derivada Stroop interferencia (I); TMT [41] para evaluar la flexibilidad cognitiva y la VP mediante el cómputo del tiempo de realización de las partes A y B del test, así como la puntuación derivada B-A libre del impacto de la velocidad [42]; y la versión del PASAT-batería neuropsicológica breve (PASAT-BNB) [43] para la evaluación de la memoria operativa y la VP mediante la presentación de una serie de 60 números (del 1 al 9) leídos en voz alta por el experimentador para que los participantes respondieran sumando el último número escuchado con el del ensayo precedente, siendo el intervalo entre estímulos determinado por el tiempo de respuesta de los participantes (se registraron el número de respuestas correctas y el tiempo total de ejecución de la prueba).

Para determinar la presencia de diferencias entre los grupos en las variables demográficas y clínicas (edad, educación, BDI, EDSS, duración de la enfermedad) se emplearon ANOVA de un factor, la prueba $t$ de Student o $\chi^{2}$. Con el fin de evitar posibles diferencias en el rendimiento entre los grupos de pacientes debidas a la medicación, se comparó el número total de fármacos con posible efecto psicomotor consumidos por los dos grupos de pacientes. Se consideraron para el análisis las siguientes categorías de medicación: antiespasmódicos y relajantes musculares, antidepresivos tricíclicos, anticolinérgicos y benzodiacepinas [26]. También se comparó el número de pacientes con EM con y sin síntomas depresivos en tratamiento inmunomodulador o inmunosupresor en el momento de la evaluación. Para la comparación del rendimiento neuropsicológico entre grupos, se realizaron ANOVA de un factor para las variables seleccionadas. El nivel de significación adoptado fue de $p<0,05$ para todos los análisis corregidos por Bonferroni para todas las comparaciones múltiples. Todos los análisis se realizaron mediante el programa estadístico SPSS v. 17.0.

\section{Resultados}

\section{Demográficos}

Dada la ausencia de puntuaciones neuropsicológicas de algunos de los pacientes, los sujetos que conformaron los tres grupos de comparación variaron en cada test (Tabla I). Por este motivo, se analizó la presencia de diferencias en género, edad y educación entre grupos de forma separada en cada una de las pruebas. Los resultados mostraron ausencia de diferencias en género entre los tres grupos considerados en la realización de las pruebas SDMT $\left(\chi_{(2)}^{2}=0,911 ; p=0,634\right)$, Stroop $\left(\chi_{(2)}^{2}=0,603 ; p=\right.$ $0,74)$, TMT $\left(\chi_{(2)}^{2}=0,778 ; p=0,678\right)$ y PASAT-BNB $\left(\chi_{(2)}^{2}=1,281 ; p=0,527\right)$. Asimismo, no se registraron diferencias en edad entre los tres grupos considerados para la comparación de las pruebas SDMT $\left(F_{(2,65)}=0,627 ; p=0,538\right)$, Stroop $\left(F_{(2,62)}=0,856\right.$; $p=0,43)$, TMT $\left(F_{(2,63)}=0,657 ; p=0,522\right)$ y PASAT$\operatorname{BNB}\left(F_{(2,63)}=0,498 ; p=0,61\right)$. No se registraron diferencias en nivel educativo entre los tres grupos comparados en la ejecución de las pruebas SDMT $\left(F_{(2,65)}=2,6 ; p=0,08\right)$, Stroop $\left(F_{(2,62)}=1,8 ; p=0,169\right)$, TMT $\left(F_{(2,63)}=1,4 ; p=0,253\right)$ y PASAT-BNB $\left(F_{(2,63)}\right.$ $=2,4 ; p=0,1)$. Sin embargo, se confirmó la presen- 
Tabla I. Características demográficas y clínicas de cada grupo de participantes. Los valores indican las puntuaciones medias (desviaciones típicas).

\begin{tabular}{|c|c|c|c|c|c|}
\hline & & SDMT & Stroop & TMT & PASAT-BNB \\
\hline & $n$ (varones) & $20(7)$ & $18(6)$ & $18(6)$ & $18(7)$ \\
\hline & Edad (años) & $43,3(7,6)$ & $43,8(7,3)$ & $43,4(6,8)$ & $43,2(8)$ \\
\hline & Educación (años) & $11,8(4)$ & $12,2(3,8)$ & $12,6(3,4)$ & $11,8(4,2)$ \\
\hline \multirow{7}{*}{$\begin{array}{l}\text { EM con } \\
\text { depresión }\end{array}$} & $\mathrm{BDI}^{\mathrm{a}}$ & $22,8(7,9)$ & $22,3(7,8)$ & $22,5(7,6)$ & $22,4(7,5)$ \\
\hline & N.ㅇ medicamentos & $0,5(0,8)$ & $0,4(0,8)$ & $0,6(0,8)$ & $0,4(0,8)$ \\
\hline & EDSS & $3,1(1,5)$ & $3,1(1,6)$ & $3,2(1,5)$ & $3,1(1,5)$ \\
\hline & $\begin{array}{l}\text { Duración de } \\
\text { la EM (meses) }\end{array}$ & $150(84,9)$ & $153,3(86,8)$ & $150,7(85,1)$ & $139,3(81,6)$ \\
\hline & $n$ (varones) & $22(5)$ & $21(5)$ & $22(5)$ & $22(5)$ \\
\hline & Edad (años) & $40,5(7,9)$ & $40,4(8,1)$ & $40,5(7,9)$ & $40,5(7,9)$ \\
\hline & Educación (años) & $13,5(3,3)$ & $13,6(3,4)$ & $13,5(3,3)$ & $13,5(3,3)$ \\
\hline \multirow{6}{*}{$\begin{array}{l}\text { EM sin } \\
\text { depresión }\end{array}$} & $\mathrm{BDI}{ }^{\mathrm{a}}$ & $7,4(3,5)$ & $7,3(3,5)$ & $7,4(3,5)$ & $7,4(3,5)$ \\
\hline & N.ㅇ medicamentos & $0,2(0,5)$ & $0,2(0,5)$ & $0,20,5)$ & $0,2(0,5)$ \\
\hline & EDSS & $2,4(1,5)$ & $2,5(1,5)$ & $2,4(1,5)$ & $2,4(1,5)$ \\
\hline & $\begin{array}{l}\text { Duración de } \\
\text { la EM (meses) }\end{array}$ & $115,1(74)$ & $116(75,7)$ & $115,1(74)$ & $115,1(74)$ \\
\hline & $n$ (varones) & $24(8)$ & $24(8)$ & $24(8)$ & $24(8)$ \\
\hline & Edad (años) & $41,5(9,1)$ & $41,5(9,1)$ & $41,5(9,1)$ & $41,5(9,1)$ \\
\hline \multirow[t]{3}{*}{ Controles } & Educación (años) & $14,6(4,7)$ & $14,6(4,7)$ & $14,6(4,7)$ & $14,6(4,7)$ \\
\hline & $\mathrm{BDI}{ }^{\mathrm{a}}$ & $5,2(3,6)$ & $5,2(3,9)$ & $5,4(3,5)$ & $5,4(3,5)$ \\
\hline & N.ㅇ medicamentos & $0,04(0,2)$ & $0,04(0,2)$ & $0,04(0,2)$ & $0,04(0,2)$ \\
\hline
\end{tabular}

BDI: inventario de depresión de Beck; EDSS: Expanded Disability Status Scale; EM: esclerosis múltiple; PASATBNB: Paced Auditory Serial Addition Test-batería neuropsicológica breve; SDMT: Symbol Digit Modality Test; TMT: Trail Making Test. a Sólo se registraron diferencias entre los tres grupos de participantes en la variable BDI, obteniendo los pacientes con EM y síntomas depresivos una puntuación mayor que los pacientes con EM sin síntomas depresivos y que los controles sanos, no diferenciándose estos últimos dos grupos entre sí.

cia de diferencias significativas en el cuestionario BDI de síntomas depresivos (SDMT: $F_{(2,65)}=69,2$; $p<0,001$; Stroop: $F_{(2,62)}=64,3 ; p<0,001$; TMT: $F_{(2,63)}=68,6 ; p<0,001$; PASAT-BNB: $F_{(2,63)}=69,1$; $p<0,001)$, obteniendo los pacientes asignados al grupo con síntomas depresivos mayores puntuaciones en comparación con los pacientes sin síntomas depresivos y los controles sanos (todos los valores de $p$ en las comparaciones post hoc fueron $<0,001$ ). No se encontraron diferencias entre los pacientes sin síntomas depresivos y los controles en relación con la puntuación en el BDI (todos los valores de $p$ en las comparaciones post hoc fueron $>0,393$ ). Dieciocho pacientes con EM sin síntomas depresivos (82\%) y 20 con EM y síntomas depresivos (90\%) estaban en tratamiento inmunomodulador/inmunosupresor en el momento de la evaluación. Los fármacos inmunomoduladores administrados fueron el interferón $\beta$-1a intramuscular o subcutáneo, el interferón $\beta$-1b y el acetato de glatiramero, mientras que el medicamento inmunosupresor fue el natalizumab. El número de pacientes tratados no difirió estadísticamente entre los grupos de pacientes con EM con y sin síntomas depresivos $\left(\chi_{(1)}^{2}=0,937\right.$; $p=0,333)$. Los dos grupos de pacientes no mostraron diferencias en ninguna de las restantes variables clínicas (todos los valores de $p$ en las comparaciones fueron $>0,249$ ) (Tabla I).

\section{Tests neuropsicológicos}

Los ANOVA de las puntuaciones Stroop-P, Stroop$\mathrm{C}$ y Stroop-PC mostraron un efecto significativo del factor grupo (Stroop-P: $F_{(2,62)}=15,8 ; p<0,001$; Stroop-C: $F_{(2,62)}=10,6 ; p<0,001$; Stroop-PC: $F_{(2,62)}$ $=6,6 ; p=0,002)$. Las pruebas post hoc mostraron diferencias de rendimiento entre los dos grupos de pacientes y los controles en todas las condiciones del test (EM sin síntomas depresivos frente a controles sanos: Stroop-P: $p=0,002$; Stroop-C: $p=$ 0,011; Stroop-PC: $p=0,035$; EM con síntomas depresivos frente a controles sanos: Stroop-P: $p<0,001$; Stroop-C: $p<0,001$; Stroop-PC: $p=0,003)$. Por el contrario, no se encontraron diferencias entre los dos grupos de pacientes en ninguna de las condiciones del test (Stroop-P: $p=0,139$; Stroop-C: $p=0,419$; Stroop-PC: $p=1)$. En contraste con los resultados mostrados en el análisis de las puntuaciones directas del test, el ANOVA realizado sobre la puntuación derivada de Stroop-I no mostró diferencias asociadas al factor grupo $\left(F_{(2,62)}=0,3 ; p<0,77\right)$ (Tabla II).

Los ANOVA de un factor sobre el tiempo de ejecución en los ensayos del TMT reveló la presencia de un efecto significativo del factor grupo tanto en el TMT-A $\left(F_{(2,63)}=8,6 ; p<0,001\right)$ como en el TMT-B $\left(F_{(2,63)}=10,1 ; p<0,001\right)$. Los análisis post hoc revelaron que los pacientes con EM y síntomas depresivos tardaron más en realizar las dos condiciones del test que los pacientes con EM sin síntomas depresivos (TMT-A: $p=0,028$; TMT-B: $p=0,008$ ) y los controles (TMT-A: $p<0,001$; TMT-B: $p<0,001$ ). 
Tabla II. Puntuaciones medias (desviación típica) y resultados de los ANOVA comparando el rendimiento neuropsicológico de los pacientes con EM con síntomas depresivos, pacientes con EM sin síntomas depresivos y controles sanos en los tests neuropsicológicos empleados.

\begin{tabular}{lcccccc}
\hline & EM con depresión & EM sin depresión & Controles & $F$ & $p$ & Post hoc \\
\hline SDMT & $29,6(10,6)$ & $40,6(13,5)$ & $48,2(8,6)$ & 15,5 & 0,001 & $a, b$ \\
\hline TMT A & $55,2(19,6)$ & $40,3(19,1)$ & $32,7(12,6)$ & 9,1 & 0,001 & $a, b$ \\
\hline TMT B & $166,8(109,9)$ & $96,5(62,3)$ & $70,1(24,2)$ & 10,1 & 0,001 & $a, b$ \\
\hline TMT B-A & $111,66(97,1)$ & $56,1(60,2)$ & $38,3(20,2)$ & 6,9 & 0,001 & $a, b$ \\
\hline Stroop-P & $87,2(17,3)$ & $98,1(15,4)$ & $114,7(17)$ & 14,5 & 0,001 & $b, c$ \\
\hline Stroop-C & $60,4(15,8)$ & $67(11,3)$ & $79(13,5)$ & 9,9 & 0,001 & $b, c$ \\
\hline Stroop-PC & $35,3(10,7)$ & $38,8(11,3)$ & $47,4(12,5)$ & 6 & 0,002 & $b, c$ \\
\hline Stroop-I & $-0,2(8,5)$ & $-0,7(7,6)$ & $-0,8(7,4)$ & 0,1 & 0,770 & - \\
\hline PASAT-BNB t & $282,3(56,5)$ & $236,5(62,7)$ & $197,5(37,5)$ & 13,3 & 0,001 & $\mathrm{a}, \mathrm{b}, \mathrm{c}$ \\
\hline PASAT-BNB C & $43,6(7,2)$ & $46,3(9,3)$ & $49,5(5,6)$ & 3,2 & 0,046 & $\mathrm{~b}$ \\
\hline
\end{tabular}

EM: esclerosis múltiple; $p$ : valor de significación asociado al efecto principal del factor grupo en los análisis de ANOVA; PASAT-BNB c: número de respuestas correctas en el Paced Auditory Serial Addition Test-batería neuropsicológica breve; PASAT-BNB t: tiempo de ejecución en el Paced Auditory Serial Addition Test-batería neuropsicológica breve; SDMT: Symbol Digit Modality Test; Stroop-C: Stroop color; Stroop-I: Stroop interferencia; Stroop-P: Stroop palabras; Stroop-PC: Stroop palabra-color; TMT: Trail Making Test. a Diferencias post hoc entre pacientes con EM sin síntomas depresivos y pacientes con EM con síntomas depresivos; ${ }^{b}$ Diferencias post hoc entre pacientes con EM con síntomas depresivos y controles; ' $\mathrm{c}$ Diferencias post hoc entre pacientes con EM sin síntomas depresivos y controles.

No se encontraron diferencias entre los pacientes con EM sin síntomas depresivos y los controles en ninguna de las dos condiciones (TMT-A: $p=0,433$; TMT-B: $p=0,622$ ). El ANOVA de un factor sobre TMT B-A reveló la presencia de un efecto significativo del factor grupo $\left(F_{(2,63)}=7,3 ; p=0,001\right)$, diferenciándose los pacientes con EM y síntomas depresivos tanto de los pacientes con EM sin síntomas depresivos $(p=0,023)$ como de los controles sanos $(p=0,001)$. Por el contrario, no se registraron diferencias significativas en la puntuación TMT B-A entre los pacientes con EM sin síntomas deprimidos y los controles $(p=0,956)$.

El ANOVA de un factor sobre el SDMT reveló la presencia de un efecto significativo del factor grupo $\left(F_{(2,65)}=15,5 ; p<0,001\right)$, obteniendo los pacientes con EM y síntomas depresivos menor número de respuestas correctas que los pacientes sin síntomas depresivos $(p=0,006)$ y los controles $(p<0,001)$. Los análisis post hoc no mostraron diferencias entre los pacientes con EM sin síntomas depresivos y los controles sanos $(p=0,073)$.

Los ANOVA de un factor realizados sobre las puntuaciones del PASAT-BNB revelaron la presen- cia de un efecto significativo del factor grupo tanto en tiempo de ejecución $\left(F_{(2,63)}=13,3 ; p<0,001\right)$ como en número de respuestas correctas $\left(F_{(2,63)}=\right.$ $3,2 ; p=0,046)$. Los análisis post hoc sobre el tiempo de ejecución revelaron que los pacientes con EM y síntomas depresivos tardaron más en realizar la tarea que los pacientes con EM sin síntomas depresivos $(p=0,024)$ y los controles $(p<0,001)$. También se evidenciaron diferencias entre los pacientes con EM sin síntomas depresivos y los controles, siendo el tiempo de ejecución más elevado en los primeros $(p=0,045)$. En cuanto al número de respuestas correctas, los análisis post hoc revelaron que los pacientes con EM y síntomas depresivos obtuvieron puntuaciones inferiores a los controles $(p=0,043)$. Ninguna otra comparación resultó significativa (todos los valores de $p$ en las comparaciones post hoc fueron $>0,456$ ) (Tabla II).

\section{Discusión}

El primer objetivo del presente estudio fue determinar el impacto de la EM en la realización de tests 
neuropsicológicos sensibles a las alteraciones de la VP. De acuerdo con los hallazgos de estudios previos, se planteó la hipótesis de que el deficiente rendimiento de los pacientes con EM no se evidenciaría de forma generalizada en los tests neuropsicológicos $[16,26]$. Los resultados de la comparación en el rendimiento neuropsicológico entre los pacientes con EM y sin síntomas depresivos y los controles sanos mostraron, por un lado, que los pacientes obtuvieron peores puntuaciones en Stroop-P, Stroop-C, Stroop-PC y PASAT-BNB (tiempo de ejecución), mientras que no se encontraron diferencias entre los grupos en el SDMT y en el TMT-A y TMT-B. Pese a que la ausencia de diferencias entre pacientes con EM sin síntomas depresivos y controles en tests de velocidad como el SDMT o el TMT parecería contradecir los estudios que sugieren la presencia de una disminución de la VP de carácter generalizado en la EM [15-18], en dichos trabajos no se controló de forma adecuada el posible impacto de la depresión [26]. De hecho, los trabajos que hasta la fecha han incluido un grupo de pacientes sin depresión no han encontrado diferencias en comparación con los controles en pruebas como el SDMT $[26,27]$, lo que apoyaría los resultados del presente estudio. Además, cabe señalar que la mayoría de los estudios anteriores han incluido muestras heterogéneas de pacientes con distintas formas clínicas de la enfermedad, lo que conlleva el riesgo de proporcionar conclusiones erróneas sobre los déficits de velocidad de un subtipo específico de pacientes [16, 32,44]. En este sentido, nuestros datos constituirían la primera evidencia sobre las dificultades de rendimiento en tests de VP de un grupo de pacientes con EMRR.

El segundo objetivo del presente trabajo fue determinar la influencia de los síntomas depresivos en el rendimiento neuropsicológico de los pacientes con EMRR. De acuerdo con los hallazgos de los estudios anteriores, se planteó la hipótesis de que los síntomas depresivos podrían desempeñar un papel en la disminución de la VP en la EM [26,31]. Los resultados mostraron que los pacientes con EMRR y síntomas depresivos realizaron peor que los pacientes sin síntomas depresivos el SDMT y el TMT. De igual forma, los pacientes con síntomas depresivos necesitaron más tiempo para finalizar la tarea en el PASAT-BNB comparados con los pacientes con EM sin síntomas depresivos, cometiendo un mayor número de errores que los controles. No se evidenciaron diferencias entre los dos grupos de pacientes en el test de Stroop. En conjunto, estos datos sugieren que los síntomas depresivos parecen afectar el rendimiento de los pacientes con EMRR en pruebas de velocidad y que su impacto varía en función del test neuropsicológico empleado. En concreto, los síntomas depresivos se perfilan como la principal variable responsable de la ejecución de los pacientes con EMRR en los tests SDMT, TMT y PASAT-BNB (número de errores), mientras que en el PASAT-BNB (tiempo de ejecución), los síntomas depresivos parecen agravar los déficits de velocidad achacables a la EM. Por último, la pobre ejecución de los pacientes con EMRR en el test de Stroop parece ser independiente de la presencia de síntomas depresivos. En general, estos resultados replican los de investigaciones previas respecto al SDMT [26] y al test de Stroop [3], consolidando la idea de que los síntomas depresivos modulan el rendimiento en el SDMT, pero no en el test de Stroop. A nuestro entender, el presente estudio representa la primera evidencia sobre el rendimiento de pacientes con EM con y sin síntomas depresivos en el TMT.

El tercer objetivo de este trabajo fue determinar si la disminución de la VP parece el principal factor que está en la base del deficiente rendimiento neuropsicológico de los pacientes con EM con y sin síntomas depresivos (relative consequence model [20]). El análisis de los resultados de los pacientes sin síntomas depresivos mostró que, pese a la presencia de diferencias en las puntuaciones directas del test de Stroop, este grupo de pacientes no mostró diferencias con los controles en la puntuación en Stroop-I. Además, estos pacientes necesitaron más tiempo para finalizar el PASAT-BNB en comparación con los controles, pese a que no cometieron un mayor número de errores. Los resultados sugieren que las diferencias entre los pacientes con EM sin síntomas depresivos y los controles sanos se debieron principalmente a un problema de velocidad. En consecuencia, y pudiéndose descartar la presencia de alteraciones específicas en otros procesos cognitivos, se puede concluir que la hipótesis del relative consequence model se mostró de utilidad para explicar el patrón de alteraciones en este grupo.

Los pacientes con síntomas depresivos no mostraron diferencias respecto a los controles sanos en la puntuación en Stroop-I. Sin embargo, sí mostraron diferencias en la puntuación en el TMT B-A con el resto de participantes. Además, los pacientes con síntomas depresivos necesitaron más tiempo para finalizar el PASAT-BNB que los otros dos grupos de participantes y cometieron un mayor número de errores que los controles. En conjunto, estos hallazgos sugieren que los déficits de velocidad por sí solos no fueron capaces de explicar el bajo rendimiento de los pacientes con síntomas depresivos en el TMT y el PASAT-BNB. Así, y pese a que la disminu- 
ción de la VP resultó un factor limitador del rendimiento cognitivo, los pacientes con EM con síntomas depresivos mostraron un déficit genuino de la flexibilidad cognitiva y la memoria operativa. A la luz de los datos, la hipótesis del relative consequence model no resultó de utilidad para explicar el rendimiento neuropsicológico de este grupo. La asunción de que los pacientes con EM deprimidos presentan un déficit de velocidad y en el funcionamiento ejecutivo es coherente con la bibliografía sobre depresión en otras poblaciones clínicas $[45,46]$.

En conclusión, los resultados de este estudio sugieren que los pacientes con EMRR sin depresión no muestran un rendimiento neuropsicológico deficitario generalizado. Asimismo, los síntomas depresivos parecen desempeñar un papel importante en la determinación de los déficits de velocidad en los pacientes con EMRR. Por último, y pese a que el impacto de la EM en las tareas neuropsicológicas parece ser explicable en términos de lentitud en el procesamiento de la información, los síntomas depresivos provocan déficits específicos en el rendimiento cognitivo diferenciables de aquéllos asociados a la velocidad. Sin duda, los presentes hallazgos constituyen un punto de partida para la caracterización de los déficits de velocidad en pacientes con diferentes cursos clínicos de la enfermedad (por ejemplo, EM primaria progresiva y EM secundaria progresiva).

\section{Bibliografía}

1. Rao SM, Leo GJ, Bernardin L, Unverzagt F. Cognitive dysfunction in multiple sclerosis. I. Frequency, patterns, and prediction. Neurology 1991; 41: 685-91.

2. Thornton AE, Raz N. Memory impairment in multiple sclerosis: a quantitative review. Neuropsychology 1997; 11: 357-66.

3. Denney DR, Sworowski LA, Lynch SG. Cognitive impairment in three subtypes of multiple sclerosis. Arch Clin Neuropsychol 2005; 20: 967-81.

4. Snyder PJ, Cappelleri JC, Archibald CJ, Fisk JD. Improved detection of differential information-processing speed deficits between two disease-course types of multiple sclerosis. Neuropsychology 2001; 15: 617-25.

5. Demaree HA, DeLuca J, Gaudino EA, Diamond BJ. Speed of information processing as a key deficit in multiple sclerosis: implications for rehabilitation. J Neurol Neurosurg Psychiatry 1999; 67: 661-3.

6. Forn C, Belenguer A, Parcet-Ibars MA, Ávila C. Informationprocessing speed is the primary deficit underlying the poor performance of multiple sclerosis patients in the Paced Auditory Serial Addition Test (PASAT). J Clin Exp Neuropsychol 2008; 30: 789-96.

7. Lengenfelder J, Bryant D, Diamond BJ, Kalmar JH, Moore NM, DeLuca J. Processing speed interacts with working memory efficiency in multiple sclerosis. Arch Clin Neuropsychol 2006; 21: 229-38.

8. Macniven JA, Davis C, Ho M, Bradshaw CM, Szabadi E, Constantinescu CS. Stroop performance in multiple sclerosis: information processing, selective attention, or executive functioning? J Int Neuropsychol Soc 2008; 14: 805-14.
9. Denney DR, Lynch SG, Parmenter BA. A 3-year longitudinal study of cognitive impairment in patients with primary progressive multiple sclerosis: speed matters. J Neurol Sci 2008; 267: 129-36.

10. Stoquart-Elsankari S, Bottin C, Roussel-Pieronne M, Godefroy O. Motor and cognitive slowing in multiple sclerosis: an attentional deficit? Clin Neurol Neurosurg 2010; 112: 226-32.

11. McGuigan C, Hutchinson M. Unrecognised symptoms of depression in a community-based population with multiple sclerosis. J Neurol 2006; 253: 219-23.

12. Nocentini U. Clinical assessment and therapy for depression. Neurol Sci 2006; 27: 341-3.

13. Tsourtos G, Thompson JC, Stough C. Evidence of an early information processing speed deficit in unipolar major depression. Psychol Med 2002; 32: 259-65.

14. Gualtieri CT, Johnson LG, Benedict KB. Neurocognition in depression: patients on and off medication versus healthy comparison subjects. J Neuropsychiatry Clin Neurosci 2006; 8: 217-25.

15. Krupp LB, Sliwinski M, Masur DM, Friedberg F, Coyle PK. Cognitive functioning and depression in patients with chronic fatigue syndrome and multiple sclerosis. Arch Neurol 1994; 51: 705-10.

16. Denney DR, Lynch, SG, Parmenter BA, Horne N. Cognitive impairment in relapsing and primary progressive multiple sclerosis: mostly a matter of speed. J Int Neuropsychol Soc 2004; 10: 948-56.

17. Lynch SG, Dickerson KJ, Denney DR. Evaluating processing speed in multiple sclerosis: a comparison of two rapid serial processing measures. Clin Neuropsychol. 2010; 24: 963-76.

18. Bodling AM, Denney DR, Lynch SG. Rapid serial processing in patients with multiple sclerosis: the role of peripheral deficits. J Int Neuropsychol Soc 2008; 14: 646-50.

19. Radloff L. The CES-D scale: a self-report depression scale for research in the general population. Applied Psychological Measurement 1977; 1: 385-401.

20. DeLuca J, Chelune GJ, Tulsky DS, Lengenfelder J, Chiaravalloti ND. Is speed of processing or working memory the primary information processing deficit in multiple sclerosis? J Clin Exp Neuropsychol 2004; 26: 550-62.

21. Grigsby J, Ayarbe S, Kravcisin N, Busenbark D. Working memory impairment among persons with chronic progressive multiple sclerosis. J Neurol 1994; 241: 125-31.

22. D’Esposito M, Onishi K, Thompson H, Robinson K, Armstrong C, Grossman M. Working memory impairments in multiple sclerosis: evidence from a dual-task paradigm. Neuropsychology 1996; 10: 51-6.

23. Archibald CJ, Fisk JD. Information processing efficiency in patients with multiple sclerosis. J Clin Exp Neuropsychol 2000; 22: 686-701.

24. Litvan I, Grafman J, Vendrell P, Martínez J, Junqué C, Vendrell J, et al. Multiple memory deficits in patients with multiple sclerosis. Exploring the working system. Arch Neurol 1988; 45: 607-10.

25. DeLuca J, Barbieri-Berger S, Johnson SK. The nature of memory impairment in multiple sclerosis: acquisition versus retrieval. J Clin Exp Neuropsychol 1994; 16: 183-9.

26. Arnett PA, Higginson CI, Voss WD, Wright B, Bender WI, Wurst JM, et al. Depressed mood in multiple sclerosis: relationship to capacity-demanding memory and attentional functioning. Neuropsychology 1999; 13: 434-46.

27. Arnett PA, Higginson CI, Voss WD, Bender, WI, Wurst, JM, Tippin JM. Depression in multiple sclerosis: relationship to working memory capacity. Neuropsychology 1999; 13: 546-56.

28. Demaree HA, Gaudino E, DeLuca J. The relationship between depressive symptoms and cognitive dysfunction in multiple sclerosis. Cogn Neuropsychiatry 2003; 8: 161-71.

29. Hartlage S, Alloy LB, Vázquez C, Dykman B. Automatic and effortful processing in depression. Psychol Bull 1993; 113: 247-78.

30. Arnett PA, Higginson CI, Randolph JJ. Depression in multiple sclerosis: relationship to planning ability. J Int Neuropsychol Soc 2001; 7: 665-74.

31. Diamond BJ, Johnson SK, Kaufman M, Graves L. Relationships 
between information processing, depression, fatigue and cognition in multiple sclerosis. Arch Clin Neuropsychol 2008; 23: 189-99.

32. Zakzanis KK. Distinct neurocognitive profiles in multiple sclerosis subtype. Arch Clin Neuropsychol 2000; 15: 115-36.

33. Polman CH, Reingold SC, Edan G, Filippi M, Hartung HP, Kappos L, et al. Diagnostic criteria for multiple sclerosis: 2005 revisions to the 'McDonald criteria'. Ann Neurol 2005; 58: 840-6.

34. Kurtzke JF. Rating neurologic impairment in multiple sclerosis: an Expanded Disability Status Scale (EDSS). Neurology 1983; 33: 1444-52.

35. Sanz J, Vázquez C. Fiabilidad, validez y datos normativos del inventario para la depresión de Beck. Psycothema 1998; 10: 303-18.

36. Beck AT, Steer RA. BDI: Beck Depression Inventory manual. New York: Psychological Corporation; 1987.

37. Lasa L, Ayuso-Mateos JL, Vázquez-Barquero JL, DíezManrique FJ, Dowrick CF. The use of the beck depression inventory to screen for depression in the general population: a preliminary analysis. J Affect Disord 2000; 57: 261-5.

38. Oldfield RC. The assessment and analysis of handedness: the Edinburgh Inventory. Neuropsychologia 1971; 9: 97-113.

39. Smith A. Symbol digit modalities test: manual. Los Angeles: Western Psychological Services; 1982.
40. Golden CJ. The Stroop Color and Word Test. Wood Dale, IL: Stoelting; 1978.

41. Reitan R, Wolfson D. The Halstead-Reitan Neuropsychological Test Battery: theory and clinical interpretation. Tucson, AZ: Neuropsychological Press; 1985.

42. Sánchez-Cubillo I, Periáñez JA, Adrover-Roig D, RodríguezSánchez JM, Ríos-Lago M, Tirapu J, et al. Construct validity of the Trail Making Test: role of task-switching, working memory, inhibition/interference control, and visuomotor abilities. J Int Neuropsychol Soc 2009; 15: 438-50.

43. Duque P, Ibáñez J, Del Barco A, Sepulcre J, De Ramón E, Fernández-Fernández O. Normalización y validación de la batería neuropsicológica breve como test neuropsicológico de referencia en la esclerosis múltiple. Rev Neurol 2012; 54 : 263-70.

44. Huijbregts SCJ, Kalkers NF, De Sonneville LMJ, De Groot V, Reuling IEW, Polman CH. Differences in cognitive impairment of relapsing remitting, secondary and primary progressive MS. Neurology 2004; 63: 335-9.

45. Veiel HOF. A preliminary profile of neuropsychological deficits associated with major depresión. J Clin Exp Neuropsychol 1997; 19: 587-603.

46. Rose EJ, Ebmeier KP. Pattern of impaired working memory during major depression. J Affect Disord 2006; 90: 149-61.

\section{Processing speed in relapsing-remitting multiple sclerosis: the role played by the depressive symptoms}

Introduction. Reduced speed of information processing seems to characterize neuropsychological performance in multiple sclerosis (MS) patients. However, the impact in speed of information processing of depressive symptoms, that are highly prevalent in this population, has not been precisely defined yet due to the presence of some methodological limitations in most preceding studies.

Subjects and methods. 42 MS remittent recurrent patients, 20 with depressive symptoms (BDI > 13), and 22 without, were compared to 24 healthy controls in neuropsychological tasks of speed of processing (SDMT, Stroop, TMT y PASAT-BNB).

Results. MS patients without depressive symptoms performed significantly worse than healthy controls the Stroop (W, C and WC), and the PASAT-BNB (execution time) tests. MS patients with depressive symptoms performed significantly worse than healthy controls the Stroop (W, C and WC), SDMT, TMT (A, B and B-A), and the PASAT-BNB (execution time and errors) tests. MS patients with depressive symptoms performed significantly worse than MS patients without depressive symptoms the SDMT, TMT (A, B and B-A), and the PASAT-BNB (execution time) tests.

Conclusions. MS impact was not generalized in neuropsychological performance of patients. Depressive symptoms seem to play and important role determining the speed deficit. While MS reduced speed of information processing, depressive symptoms were associated to specific cognitive deficit different from speed ones.

Key words. Attention. Depression. Executive functions. Multiple sclerosis. Neuropsychological assessment. Speed of processing. 